\title{
Efeitos da blefaroplastia na síndrome da disfunção lacrimal
}

\section{Effects of blepharoplasty at the dysfunctional tear syndrome}

Juliana Silverio', Lucia Miriam Dumont Lucci², Nilson Lopes da Fonseca Júnior³ ${ }^{3}$ José Ricardo Carvalho Lima Rehder ${ }^{4}$

\section{ReSUMO}

Objetivo: Avaliar a presença da síndrome da disfunção lacrimal e alterações na sensibilidade corneana no pré e pósoperatório de pacientes com indicação de blefaroplastia superior. Métodos: Foram avaliados 30 pacientes com indicação cirúrgica para correção de dermatocálase em pálpebra superior de ambos os olhos. Obtida história clínica e realizado exame oftalmológico geral, incluindo exames como teste de Schirmer I, coloração com Lissamina Verde, tempo de ruptura do filme lacrimal, coloração com fluoresceína e teste da sensibilidade corneana com estesiômetro de Cochet-Bonnet, além de teste subjetivo pelo questionário ODSI. Os exames foram realizados antes e após 90 dias da cirurgia. Resultados: Apenas 23 pacientes completaram o seguimento, sendo o sexo feminino predominante. A idade dos pacientes variou de 63 a 87 anos. De acordo com o questionário ODSI aplicado, observou-se que houve melhora dos sintomas, mas a análise estatística mostrou um resultado não-significante. Com relação à análise dos resultados obtidos com o estesiômetro de Cochet-Bonnet, 7 pacientes apresentaram uma melhora na sensibilidade corneana, 9 pacientes diminuíram a resposta e 7 mantiveram seus valores. Os resultados do BUT lacrimal, colorações com Lissamina verde e Fluoresceína foram considerados estatisticamente não-significantes. O teste de Schirmer I demonstrou uma melhora estatisticamente significativa da produção lacrimal no pós-operatório. Conclusão: Neste trabalho observou-se que os pacientes submetidos à blefaroplastia superior apresentaram uma melhora nos sinais e sintomas relacionados à SDL, apesar de ter sido diagnosticado uma diminuição na sensibilidade corneana não estatisticamente significante.

Descritores: Blefaroplastia; Síndromes do olho seco; Pálpebras/fisiopatologia

\section{Abstract}

Purpose: To evaluate the presence of dysfunctional tear syndrome and changes in corneal sensitivity in the preoperative and postoperative patients undergoing upper blepharoplasty. Methods: Thirty patients with surgical indication for correction of upper eyelid dermatochalasis in both eyes. Obtained clinical history and general ophthalmologic examination, including tests such as Schirmer's test, Lissamine Green staining, tear break-up time and fluorescein staining, corneal sensitivity was evaluated using the Cochet-Bonnet esthesiometer, and subjective test by questionnaire ODSI. The examinations were performed before and after 90 days of surgery. Results: Only 23 patients completed the follow-up, with females predominant. The patients' ages ranged from 63 to 87 years. According to the questionnaire ODSI applied, we found that symptoms improved, but statistical analysis showed a nonsignificant result. Regarding the analysis of results obtained with the Cochet-Bonnet esthesiometer, 7 patients showed an improvement in corneal sensitivity, nine patients declined to answer and 7 maintained their values. The results of the tear BUT, and stained with Fluorescein and Lissamine green were considered statistically non-significant. The Schirmer's test showed a statistically significant improvement in tear production in the postoperative period. Conclusion: In this study we observed that patients undergoing upper blepharoplasty showed an improvement in the signs and symptoms related to $S D L$, despite having been diagnosed with a decrease in corneal sensitivity, not statistically significant.

Keywords: Blepharoplasty; Dry eye syndromes; Eyelids/physiopathology

\footnotetext{
${ }^{1}$ Médica Colaboradora do Setor de Plástica Ocular do Departamento de Oftalmologia da Faculdade de Medicina do ABC - FMABC Santo André (SP) - Brasil;

${ }^{2}$ Doutora; Médica Colaboradora do Setor de Plástica Ocular do Departamento de Oftalmologia da Faculdade de Medicina do ABC FMABC - Santo André (SP) - Brasil;

${ }^{3}$ Mestre; Chefe dos Setores de Plástica Ocular e Órbita do Departamento de Oftalmologia da Faculdade de Medicina do ABC -

FMABC - Santo André (SP) - Brasil;

${ }^{4}$ Professor Titular e Chefe do Departamento de Oftalmologia da Faculdade de Medicina do ABC - FMABC - Santo André (SP) - Brasil. Trabalho realizado no Ambulatório de Plástica Ocular da Disciplina de Oftalmologia da Faculdade de Medicina do ABC - FMABC Santo André (SP), Brasil.
}

Os autores declaram inexistir conflitos de interesse.

Recebido para publicação em: 20/10/2010 - Aceito para publicação em 16/2/2011 


\section{INTRODUÇÃO}

A síndrome da disfunção lacrimal (SDL), conhe cida como olho seco, tem uma alta prevalência, atingindo de 14 a $33 \%$ da população mundi$\mathrm{al}^{(1)}$. Caracterizada por uma desordem multifatorial lacrimal e da superfície ocular, associada com sintomas de desconforto ou distúrbios visuais ${ }^{(2)}$. Estes sintomas incluem ardor, prurido, hiperemia conjuntival, lacrimejamento, sensação de corpo estranho e fotofobia ${ }^{(3)}$.

Blefaroplastia é uma das cirurgias estéticas mais realizadas no mundo e o olho seco é uma das complicações reconhecidas e temidas pelos cirurgiões neste tipo de procedimento, com uma incidência de 8 a $21 \%{ }^{(4-6)}$. Existem muitos fatores responsáveis pelo olho seco após a blefaroplastia como lagoftalmo, retração palpebral, diminuição do reflexo de piscar e alterações do filme lacrimal, resultando em um aumento da evaporação lacrimal e sintomas da SDL ${ }^{(3-7)}$. Por isso é de extrema importância uma avaliação detalhada no pré-operatório observando as condições pré-existentes que levem a anormalidades do filme lacrimal que possam aumentar o risco do paciente apresentar complicações no pós-operatório ${ }^{(8)}$

Estudos anteriores demonstraram que a sensibilidade da pálpebra superior pode ser temporariamente afetada após a blefaroplastia ${ }^{(9)}$. Isto é devido a uma interrupção dos ramos sensoriais do nervo trigêmeo durante a incisão e na dissecção realizada na pálpebra. Ramos do trigêmeo também são responsáveis pela sensibilidade na superfície ocular e são importantes na estimulação da glândula lacrimal a na produção lacrimal ${ }^{(10-12)}$.

O sistema lacrimal secretor consiste em dois grupos distintos: o basal e o reflexo. Os secretores basais são as glândulas de Meibomius da placa tarsal, as células caliciformes e as glândulas lacrimais acessórias conjuntivais. Em condições normais, todo o volume lacrimal produzido pelos secretores basais evapora da superfície corneana. Os secretores reflexos respondem, quando estimulados por estas mudanças, secretando mais lágrima. Eles incluem a glândula lacrimal principal e as glândulas lacrimais acessórias ${ }^{(13)}$.

O filme lacrimal é composto pelas camadas mucínica, aquosa e lipídica. A camada mucínica consiste em glicoproteínas de alto peso molecular que se aderem ao epitélio e formam uma superfície hidrofílica ${ }^{(14)}$. A camada aquosa constitui o maior volume da lágrima e fica acima da camada de mucina. Por último, as glândulas de Meibomius produzem a camada lipídica, que é responsável pela estabilização do filme aumentando a tensão superficial e diminuindo a evaporação(15).

O presente estudo tem por objetivo avaliar a presença da síndrome da disfunção lacrimal e alterações na sensibilidade corneana no pré e pós-operatório de pacientes com indicação de blefaroplastia superior.

\section{Métodos}

Estudo prospectivo, aprovado pelo Comitê de Ética em Pesquisa da Faculdade de Medicina do ABC, onde foram avaliados 30 pacientes do ambulatório de Plástica Ocular da Disciplina de Oftalmologia da Faculdade de Medicina do ABC, com indicação cirúrgica para correção de dermatocálase em pálpebra superior de ambos os olhos, que não possuíam antecedentes de doenças palpebrais ou corneanas cicatriciais, ou faziam uso de próteses oculares.

A avaliação pré-operatória foi baseada na história clínica e exame oftalmológico geral, incluindo exames objetivos como teste de Schirmer I, coloração com Lissamina Verde, tempo de ruptura do filme lacrimal $($ Break-up-time $=$ BUT $)$, coloração com fluoresceína e teste da sensibilidade corneana com estesiômetro de Cochet-Bonnet (Luneau, França), além de teste subjetivo pelo questionário ODSI (Ocular Surface Disease Index ${ }^{(16)}$. No teste de Schirmer I (sem anestesia), para medir a secreção basal e reflexa, foi considerado normal valores acima de $10 \mathrm{~mm}$ em 5 minutos. O teste com coloração de Lissamina Verde (bastão de papel umidificado com lágrimas artificiais) foi graduado em pontos de acordo com a escala de Van Bijsterveld's' ${ }^{(17)}$, utilizada na coloração com Rosa Bengala. O BUT foi considerado normal acima de 5 segundos e o teste de fluoresceína também graduado em pontos de acordo com o critério proposto por Shimmura e colaboradores ${ }^{(18)}$, anormal acima de 1 . A sensibilidade corneana foi medida com o estesiômetro expondo-se o filamento de nylon totalmente $(6 \mathrm{~cm})$ e aplicando perpendicularmente à córnea. O filamento era então diminuído $0,5 \mathrm{~cm}$ até a primeira resposta, como o piscar. O comprimento máximo do filamento necessário para produzir a primeira resposta foi registrado como a sensibilidade corneana.

Todos os pacientes foram submetidos ao procedimento cirúrgico. Feita a demarcação prévia, respeitando-se o limite de 10 milímetros da margem palpebral e do supercílio e após anestesia local com infiltração no subcutâneo com lidocaína 1\% e epinefrina 1:100.000, realizou-se a excisão de pele e de uma faixa de músculo orbicular através de uma incisão na pele com bisturi lâmina $\mathrm{n}^{\circ} 15$. A incisão cutânea foi suturada utilizando 
fio mononylon 6.0 (Nylon ${ }^{\circledR} 6.0$, agulha $1,5 \mathrm{~cm}$, Ethicon, Somerville, NJ,EUA) com pontos simples separados.

Ao receber alta hospitalar, o paciente era orientado a realizar compressas frias sobre a região operada, sendo prescrito antibiótico e anti-inflamatório, via oral, durante a primeira semana após retirada dos pontos. Os pacientes foram reavaliados e submetidos aos mesmos exames realizados na avaliação pré-operatória após três meses.

\section{Resultados}

Dos 30 pacientes que iniciaram o acompanhamento, quatro tiveram a cirurgia suspensa por hipertensão arterial sistêmica (HAS) descontrolada e outros três, submetidos à cirurgia de blefaroplastia, perderam seguimento e não retornaram para a avaliação após os três meses.

Foi utilizado o programa SPSS (Statistical Package for Social Sciences), versão 17.0, para a análise dos resultados. Adotou-se o nível de significância de 5\%, para a aplicação dos testes estatísticos, ou seja, quando o valor da significância calculada (p) for menor do que $5 \%$ observa-se uma diferença estatisticamente significante, e quando o valor da significância calculada (p) for igual ou maior do que $5 \%$ observa-se uma diferença estatisticamente não-significante.

Dentre os 23 pacientes avaliados quatro $(17,39 \%)$ eram do sexo masculino e dezenove $(82,61 \%)$ do sexo feminino. A idade dos pacientes variou de 63 a 87 anos (média 69,56). Todos os pacientes eram da raça branca. As patologias prévias encontradas foram HAS em dez pacientes (43,47\%), diabetes mellitus tipo II em quatro pacientes $(17,39 \%)$ e glaucoma em um paciente $(4,34 \%)$. Ao exame biomicroscópico foi constatado que onze pacientes $(47,82 \%)$ apresentavam blefarite e quatro $(17,39 \%)$ pterígio.

Sensação de peso nos olhos, devido ao excesso de pele da pálpebra superior foi referida por vinte pacientes $(86,95 \%)$, enquanto que os sintomas relacionados à SDL, como: irritação, lacrimejamento, dor, hiperemia conjuntival e desconforto com o vento foram referidos por cinco $(21,34 \%)$. Estes cinco pacientes já faziam uso de lubrificantes oculares no pré-operatório e no pós; três $(13,04 \%)$ mantiveram a forma de aplicação; um (4,34\%) deixou de usar e um aumentou a frequência. Apenas um paciente que não usava lubrificante iniciou seu uso após a cirurgia. De acordo com o questionário ODSI aplicado (Tabela 1), observou-se que houve melhora dos sintomas, mas a análise estatística mostrou um resultado nãosignificante.

Com relação à análise dos resultados obtidos com o estesiômetro de Cochet-Bonnet, sete pacientes (30,43\%) apresentaram uma melhora na sensibilidade corneana, nove pacientes $(39,13 \%)$ diminuíram a resposta e sete $(30,43 \%)$ mantiveram seus valores (Tabela 2$)$.

Os resultados do BUT lacrimal e colorações com lissamina verde e fluoresceína encontram-se na Tabela 3 , e foram considerados estatisticamente nãosignificantes.

O teste de Schirmer I demonstrou uma melhora estatisticamente significativa da produção lacrimal com uma média de 15,70 ( $\pm 10,38)$ no pré-operatório e de

Tabela 1

Resultados obtidos pelo questionário OSDI, pré e pós-operatório

\begin{tabular}{cccccccccc}
\hline OSDI & $\mathbf{n}$ & Média & Desvio padrão & Mínimo & Máximo & Percentil 25 & Mediana & Percentil 75 & Valor de p \\
\hline Pré & 23 & 37,89 & 18,71 & 0,00 & 66,66 & 25,00 & 44,44 & 52,50 & 0,702 \\
Pós & 23 & 35,06 & 18,15 & 0,00 & 63,00 & 22,50 & 32,50 & 55,55 & \\
\hline
\end{tabular}

Tabela 2

Sensibilidade corneana pré e pós-operatória

\begin{tabular}{ccccc}
\hline $\begin{array}{c}\text { Sensibilidade } \\
\text { corneana }\end{array}$ & Média & $\begin{array}{c}\text { Desvio } \\
\text { padrão }\end{array}$ & Mediana & $\begin{array}{c}\text { Significância } \\
\text { Valor de p }\end{array}$ \\
\hline pré-operatória OD & 50,87 & 12,49 & 55,00 & 0,298 \\
pós-operatória OD & 46,52 & 17,87 & 55,00 & \\
pré-operatória OE & 51,30 & 12,72 & 55,00 & 0,168 \\
pós-operatória OE & 46,74 & 16,83 & 55,00 & \\
\hline
\end{tabular}


Tabela 3

Resultados pré e pós-operatório do BUT lacrimal e colorações com lissamina verde e fluoresceína

\begin{tabular}{lcccccc}
\hline & Média BUT & Valor de $\mathbf{p}$ & Média Lissamina verde & Valor de p & Média Fluoresceína & Valor de p \\
\hline pré-operatório OD & $5,61( \pm 2,50)$ & 0,476 & $2,57( \pm 1,27)$ & 0,439 & $0,61( \pm 1,03)$ & 0,916 \\
pós-operatório OD & $6,00( \pm 2,54)$ & & $2,26( \pm 1,60)$ & & $0,61( \pm 0,89)$ & \\
pré-operatório OE & $5,61( \pm 2,97)$ & 0,954 & $2,61( \pm 1,62)$ & 0,664 & $0,39( \pm 0,78)$ & 0,916 \\
pós-operatório OE & $5,57( \pm 2,68)$ & & $2,39( \pm 1,50)$ & & $0,39( \pm 0,72)$ & \\
\hline
\end{tabular}

21,09 $( \pm 12,92)$ no pós-operatório $(p=0,021)$.

Durante o estudo não ocorreram complicações decorrentes da cirurgia.

\section{DısCUSSÃO}

De uma forma geral houve uma melhora dos sinais e sintomas dos pacientes após a cirurgia palpebral. Os critérios que apresentaram melhora foram a coloração com Lissamina verde, o índice OSDI e o teste de Schirmer I, sendo este último estatisticamente significante. $\mathrm{O}$ aparecimento da SDL ou a piora do quadro pré-operatório podem ocorrer em pacientes que são submetidos a uma blefaroplastia mais agressiva. Isto provavelmente resulta de lagoftalmo, retração palpebral, insuficiência da bomba lacrimal, ou uma combinação destes fatores. A integridade do músculo orbicular e sua inervação pós-operatória têm um importante papel em todas estas condições que podem resultar em um quadro de olho seco ${ }^{(19)}$.

Alguns pacientes com dermatocálase apresentam sintomas similares aos portadores de ceratoconjuntivite $\mathrm{seca}^{(20)}$. Indivíduos com uma pálpebra superior volumosa tendem a sobrecarregar os músculos frontal e levantador da pálpebra, elevando a sobrancelha e acentuando os sulcos na região frontal, na tentativa de melhorar a diminuição de campo visual superior causada pelas pálpebras pesadas, podendo levar a uma fadiga periorbitária. Esta fadiga interfere no mecanismo de piscar, aumentando a evaporação do filme lacrimal, levando a uma superfície ocular relativamente seca ${ }^{(6)}$. Outros fatores que contribuem para estes sintomas são a blefarite e a presença de pterígio. Neste estudo onze pacientes $(47,82 \%)$ possuíam blefarite e quatro $(17,39 \%)$ pterígio, porém nem todos apresentavam queixas.

A SDL pode ter um impacto significativo na qualidade visual dos pacientes, sendo relatadas dificuldades com o uso de computadores, com a leitura e dirigir à noite $^{(21)}$. Atualmente cerca de $12 \%$ dos homens e $17 \%$ das mulheres na população geral apresentam quadro de ressecamento ocular ${ }^{(22)}$. A lágrima é composta de três camadas (mucínica, aquosa e lipídica) produzidas por glândulas presentes na conjuntiva limbar, bulbar e tarsal e na margem palpebral, além da glândula lacrimal principal. O filme lacrimal é restabelecido entre as piscadas e sua estabilidade depende de uma produção normal, um fechamento palpebral completo e uma frequência de piscar adequada ${ }^{(20,23)}$. Modificações cirúrgicas da anatomia palpebral podem alterar o fechamento palpebral e o mecanismo de piscar. Existem diversos métodos para se aperfeiçoar uma cirurgia estética, no entanto abordagens menos agressivas, como a retirada de uma fina faixa de músculo orbicular e a exérese conservadora tanto de pele quanto de gordura, podem evitar muitas complicações desnecessárias $^{(8)}$.

A quemose é um fator que intensifica o desconforto ocular e os sintomas relacionados com a SDL no pós-operatório. A menor intensidade da inflamação obtida com uma dissecção mais cuidadosa evitando-se lesar as inervações e, consequentemente, diminuindo o trauma, pode contribuir para uma quemose menos intensa ${ }^{(24)}$.

A literatura é controvérsia com relação à produção da lágrima após a blefaroplastia. Alguns autores ${ }^{(25-}$ ${ }^{27)}$ observaram diminuição na produção da lágrima e outros $^{(28-30)}$, incluindo o presente estudo, observaram um aumento, mostrado pelo resultado no teste de Schirmer no pós-operatório. Ainda que se desconheça tal razão, Kim et al. ${ }^{(28)}$ procuram explicar este resultado com a possibilidade de uma reinervação palpebral (supraorbital e supratroclear) pós-operatória que estimularia a divisão oftálmica do $\mathrm{V}$ par craniano, afetando desta maneira o nervo lacrimal. Como consequência os mecanismos de autorregulação lacrimal poderiam ser alterados.

Pacientes com um quadro de olho seco possuem sintomas de irritação devido a uma deterioração da superfície ocular. No entanto, a percepção da dor é paradoxalmente menor nestes pacientes. Do ponto de vista fisiológico os nervos sensórios podem se adaptar devido a uma estimulação, sendo que a frequência e a intensidade das ações podem levar potencialmente a uma adap- 
tação. O processo envolve uma simples acomodação dos axônios ${ }^{(31)}$. Erosões prolongadas e irritações na superfície ocular podem elevar o limiar da dor, desta maneira estímulos mais intensos são necessários para se obter a sensação corneana em pacientes com olho seco ${ }^{(26)}$.

Neste estudo observou-se uma diminuição na sensibilidade corneana de uma forma geral, o que corrobora com os resultados de Xu et al. e Rees \& LaTrenta ${ }^{(26,30)}$, apesar de estatisticamente não-significantes. A sensibilidade corneana é importante na manutenção da integridade do epitélio corneano ${ }^{(32)}$ e da produção lacrimal normal através da estimulação trigeminal ${ }^{(26)}$. A inervação corneana é derivada da divisão oftálmica do quinto par craniano (trigêmeo), através dos nervos ciliares longos. As fibras nervosas corneanas possuem dois tipos de terminações: numerosos filamentos entre as lamelas estromais (plexo subepitelial) e através da membrana de Bowman, atingindo o epitélio (plexo intraepitelial $)^{(33)}$. O mecanismo exato de como a sensibilidade corneana é afetada após uma cirurgia palpebral é incerto. Alguns autores não acreditam que a incisão e dissecção palpebral sejam capazes de romper ou traumatizar os ramos do nervo ciliar responsável pela sensibilidade da superfície ocular ${ }^{(10,11,34)}$. No entanto, a sensibilidade corneana pode ser modulada por outros fatores além da inervação sensorial, como o aumento da concentração de citocinas inflamatórias e peptídeos opióides num quadro inflamatório ${ }^{(27,35)}$. É possível que a cirurgia palpebral e a inflamação pós-operatória esperada também resultem em um aumento da secreção de mediadores inflamatórios e por isso haja uma diminuição da sensibilidade corneana ${ }^{(28)}$.

Os resultados do teste de Schirmer I não se correlacionam com a sensibilidade corneana. A ausência de correlação entre a produção lacrimal e a sensibilidade corneana pode ser atribuída a uma menor confiabilidade nos testes utilizados ou ao fato de a diminuição da sensibilidade corneana ser independente da produção reflexa lacrimal ${ }^{(26)}$.

Embora a maioria dos achados clínicos objetivos não tenha apresentado uma melhora significativa após a blefaroplastia, os sintomas oculares subjetivos melhoraram. Acreditamos ser devido a uma diminuição da reação inflamatória, uma melhora no campo visual superior e a uma redução do uso dos músculos frontal e levantador da pálpebra. Outra razão pode ser que a diminuição do tônus destes músculos leve a uma melhora na frequência de piscar, o que ajuda na troca do filme lacrimal ${ }^{(29)}$. No entanto Abell et al. ${ }^{(23)}$ não relacionam a blefaroplastia a alterações no mecanismo de piscar.

A blefaroplastia superior é o procedimento cirúr- gico cosmético mais comumente realizado tanto em homens como em mulheres. Pacientes no pós-operatório de uma cirurgia estética encontram-se mais seguros em relação à sua aparência. Este fator psicológico deve ter uma influência nos sintomas oculares subjetivos ${ }^{(20,29)}$.

\section{Conclusão}

Neste trabalho observou-se que os pacientes submetidos à blefaroplastia superior apresentaram uma melhora nos sinais e sintomas relacionados à SDL, apesar de ter sido diagnosticado uma diminuição na sensibilidade corneana não estatisticamente significante.

\section{REFERÊNCIAS}

1. Behrens A, Doyle JJ, Stern L, Chuck RS, McDonnell PJ, Azar DT, Dua HS, Hom M, Karpecki PM, Laibson PR, Lemp MA, Meisler DM, Del Castillo JM, O'Brien TP, Pflugfelder SC, Rolando M, Schein OD, Seitz B, Tseng SC, van Setten G, Wilson SE, Yiu SC; Dysfunctional tear syndrome study group. Dysfunctional tear syndrome: a Delphi approach to treatment recommendations. Cornea. 2006;25(8):900-7. Comment in: Cornea. 2007;26(7):901.

2. The definition and classification of dry eye disease: report of the Definition and Classification Subcommittee of the International Dry Eye WorkShop (2007). Ocul Surf. 2007;5(2):75-92.

3. Korn BS, Kikkawa DO, Schanzlin DJ. Blepharoplasty in the post-laser in situ keratomileusis patient: preoperative considerations to avoid dry eye syndrome. Plast Reconstr Surg. 2007;119(7):2232-9. Comment in: Plast Reconstr Surg. 2008;121(4):1506-7; author reply 1507.

4. Swartz RM, Schultz RC, Seaton JR. "Dry eye" following blepharoplasty. Cause or coincidence? Plast Reconstr Surg. 1974;54(6):644-7.

5. Morax S, Touitou V. Complications of blepharoplasty. Orbit. 2006;25(4):303-18. Review.

6. Vold SD, Carroll RP, Nelson JD. Dermatochalasis and dry eye. Am J Ophthalmol. 1993;115(2):216-20.

7. Graham WP 3rd, Messner KH, Miller SH. Keratoconjunctivitis sicca symptoms appearing after blepharoplasty. The "dry eye" syndrome. Plast Reconstr Surg. 1976;57(1):57-61.

8. Hamawy AH, Farkas JP, Fagien S, Rohrich RJ. Preventing and managing dry eyes after periorbital surgery: a retrospective review. Plast Reconstr Surg. 2009;123(1):353-9.

9. Black EH, Gladstone GJ, Nesi FA. Eyelid sensation after supratarsal lid crease incision. Ophthal Plast Reconstr Surg. 2002;18(1):45-9.

10. Belmont C, Acosta MC, Gallar J. Neural basis of sensation in intact and injured corneas. Exp Eye Res. 2004;78(3):513-25.

11. Müller LJ, Vrensen GF, Pels L, Cardozo BN, Willekens B. Architecture of human corneal nerves. Invest Ophthalmol Vis Sci. 1997;38(5):985-94.

12. Kanellopoulos AJ, Pallikaris IG, Donnenfeld ED, Detorakis S, Koufala K, Perry HD. Comparison of corneal sensation following photorefractive keratectomy and laser in situ keratomileusis. J Cataract Refract Surg. 1997;23(1):34-8. 
13. McKinney P, Zukowski ML. The value of tear film breakup and Schirmer's tests in preoperative blepharoplasty evaluation. Plast Reconstr Surg. 1989;84(4):572-6; discussion 577.

14. Argüeso P, Gipson IK. Epithelial mucins of the ocular surface: structure, biosynthesis and function. Exp Eye Res. 2001;73(3):281-9.

15. Rolando $\mathrm{M}$, Zierhut $\mathrm{M}$. The ocular surface and tear film and their dysfunction in dry eye disease. Surv Ophthalmol. 2001;45 Suppl 2:S203-10.

16. Schiffman RM, Christianson MD, Jacobsen G, Hirsch JD, Reis BL. Reliability and validity of the Ocular Surface Disease Index. Arch Ophthalmol. 2000;118(5):615-21. Comment in: Arch Ophthalmol. 2001;119(3):456.

17. van Bijsterveld OP. Diagnostic tests in Sicca syndrome. Arch Ophthalmol. 1969;82(1):10-4.

18. Shimmura S, Ono M, Shinozaki K, Toda I, Takamura E, Mashima Y, Tsubota K. Sodium hyaluronate eyedrops in treatment of dry eyes. Br J Ophthalmol. 1995;79(11):1007-11.

19. Saadat D, Dresner SC. Safety of blepharoplasty in patients with preoperative dry eyes. Arch Facial Plast Surg. 2004;6(2):101-4.

20. Lima CGMG, Siqueira GB, Cardoso IH, Sant'Anna AEB, Osaki MH. Avaliação do olho seco no pré e pós-operatório da blefaroplastia. Arq Bras Oftalmol. 2006;69(2):227-32.

21. Nelson JD, Helms H, Fiscella R, Southwell Y, Hirsch JD. A new look at dry eye disease and its treatment. Adv Ther. 2000;17(2):84-93.

22. Moss SE, Klein R, Klein BE. Prevalence of and risk factors for dry eye syndrome. Arch Ophthalmol. 2000;118(9):1264-8.

23. Abell KM, Cowen DE, Baker RS, Porter JD. Eyelid kinematics following blepharoplasty. Ophthal Plast Reconstr Surg. 1999;15(4):236-42.

24. Enzer YR, Shorr N. Medical and surgical management of chemosis after blepharoplasty. Ophthal Plast Reconstr Surg. 1994;10(1):57-63.

25. Rees TD, Jelks GW. Blepharoplasty and the dry-eye syndrome: guidelines for surgery? Plast Reconstr Surg. 1981;68(2):249-52.
26. Xu KP, Yagi Y, Tsubota K. Decrease in corneal sensitivity and change in tear function in dry eye. Cornea. 1996;15(3):235-9.

27. Yen MT, Pflugfelder SC, Feuer WJ. The effect of punctal occlusion on tear production, tear clearance, and ocular surface sensation in normal subjects. Am J Ophthalmol. 2001;131(3):314-23.

28. Kim HH, De Paiva CS, Yen MT. Effects of upper eyelid blepharoplasty on ocular surface and tear production. Can J Ophthalmol. 2007;42(5):739-42.

29. Floegel I, Horwath-Winter J, Muellner K, Haller-Schober EM. A conservative blepharoplasty may be a means of alleviating dry eye symptoms. 2003; Acta Ophthalmol Scand. 2003;81(3):230-2

30. Rees TD, LaTrenta GS. The role of the Schirmer's test and orbital morphology in predicting dry-eye syndrome after blefaroplasty. Plast Reconstr Surg. 1988;82(4):619-25.

31. Ganong WF. Initiation of impulse in sense organs. In: Ganong WF, editor. Review of medical physiology. 8th ed. Stamford, Conn: Appleton \& Lange; 1977. p. 63.

32. Martin XY, Safran AB. Corneal hypoesthesia. Surv Ophthalmol. 1988;33(1):28-40. Review.

33. Forester JV, Dick AD, McMenamin PG, Lee WR. The eye: basic sciences in practice. 2nd ed. Philadelphia: Saunders; 2002. p. 20.

34. Scott KR, Tse DT, Kronish JW. Vertically oriented upper eyelid nerve fibers. A clinical, anatomical and immunohistochemical study. Ophthalmology. 1992;99(2):222-6.

35. Afonso AA, Monroy D, Stern ME, Feuer WJ, Tseng SC, Pflugfelder SC. Correlation of tear fluorescein clearance and Schirmer test scores with ocular irritation symptoms. Ophthalmology. 1999;106(4):803-10
Endereço para correspondência:
Juliana Silverio
Rua Álvaro Rodrigues, $\mathrm{n}^{\circ} 792$
CEP 04582-001 - São Paulo (SP), Brasil
Fax (11) 55318042
e-mail: julianasilverio@yahoo.com.br 